\title{
New Cytotoxic Cycloartane Triterpenes from the Aerial Parts of Actaea heracleifolia (syn. Cimicifuga heracleifolia)
}

\author{
Authors \\ Affiliations \\ 1 State Key Laboratory of Phytochemistry and Plant \\ Resources in West China, Kunming Institute of Botany, \\ Chinese Academy of Science, Kunming, China \\ 2 University of the Chinese Academy of Science, Beijing, \\ China \\ 3 Yunnan Key Laboratory of Natural Medicinal Chemistry \\ Chinese Academy of Sciences, Kunming, China \\ Key words \\ Actaea heracleifolia, Ranunculaceae, 15,16-seco-cycloartane \\ triterpene, cycloartane triterpene glycosides, cytotoxicity, \\ NMR \\ received June 25, 2018 \\ revised August 21, 2018 \\ accepted August 28, 2018 \\ Bibliography \\ DOI https://doi.org/10.1055/a-0733-7229 \\ Published online September 19, 2018 | Planta Med 2019; 85: \\ 154-159 @ Georg Thieme Verlag KG Stuttgart · New York | \\ ISSN 0032-0943
}

Qiang-Qiang Shi ${ }^{1,2,3 *}$, Wei-Hua Wang ${ }^{1,2,3 *}$, Jing Lu $^{1,2,3}$, Da-Shan Li $^{1,3}$, Lin Zhou ${ }^{1,3}$, Ming-Hua Qiu ${ }^{1,3}$

\section{Correspondence}

Prof. Ming-Hua Qiu

State Key Laboratory of Phytochemistry and Plant Resources in West China, Kunming Institute of Botany, Chinese Academy of Science

132 Lanhei Road, Kunming 650201, China

Phone: + 8687165223325 , Fax: + 8687165223327

mhchiu@mail.kib.ac.cn

\section{Supporting information available online at}

http://www.thieme-connect.de/products

\section{ABSTRACT}

One new 15,16-seco-cycloartane triterpene (1), three new cycloartane triterpene glycosides (2-4), and five known compounds (5-9) were isolated from the aerial parts of Actaea heracleifolia. The chemical structures of these compounds were determined on the basis of NMR analysis, HRTOF-ESIMS data, and other spectroscopic methods. Selected compounds were evaluated for their cytotoxicity against human tumor cell lines (HL-60, SMMC-7721, A549, MCF-7, and SW480) in vitro. Compounds 3 and 4 showed weak activity against the $\mathrm{HL}-60, \mathrm{~A}-549$, and MCF-7 cell lines with $\mathrm{IC}_{50}$ values ranging from 21.34 to $36.98 \mu \mathrm{M}$.

\section{Introduction}

The Actaea species, belonging to the family Ranunculaceae, has a long history of uses as a medicinal herb worldwide. In China, the rhizomes of Actaea heracleifolia Kom., Actaea dahurica (Turcz.) Maxim., and Actaea foetida L., officially listed in the Chinese Pharmacopoeia with the name "shengma", are used as cooling and detoxifying agents for the treatment of headache, sore throat, and toothache [1]. In Europe and the United States, Actaea racemosa (L.) Nutt., commonly called black cohosh, is also used as a dietary supplement for women's health during climacteric periods [2,3]. Triterpene glycosides have been considered the main active components of Actaea species, and phytochemical investigations of plant species led to the isolation of a series of 9,19cycloartane triterpenes characterized with unique structural features [4], including ring A opened cycloartane triterpenes, such as 3,4-seco-4-hydroxy-3-cimigenolate [5]; ring $A$ expanded cimigenol-type triterpenes, such as cimiheraclein A [6]; 15,16-seco- cycloartane triterpenes [5-10]; ring B opened triterpenes [6, 11, 12]; and cycloartane triterpenes with new skeletons, such as cimicifugadine [13], cimicifine B [14], and cimyunnins A-D [15]. In a further investigation of the aerial parts of $A$. heracleifolia, we found four new cycloartane triterpenes (1-4) and five known ones: cimiheraclein $D(5)$ [6], 25-anhydrocimigenol-3-O- $\alpha$-L-arabinopyrano-

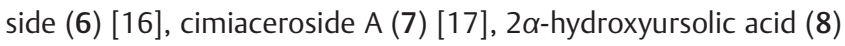
[18], and $1 \alpha, 3 \beta, 19 \alpha, 23$-tetrahydroxyurs-12-en-28-oic acid-28-O$\beta$-D-xylopyranoside (9) [19] ( $\bullet$ Fig. 1). Compound 1 was a D ringcleaved 15,16-seco-cycloartane triterpene. Compounds $2-4$ were 9,19-cycloartane triterpenoid saponins with a fused monosaccharide moiety. Herein, we reported the structure determination by $1 \mathrm{D} / 2 \mathrm{D}$ NMR analysis of the new compounds and the evaluation of the cytotoxic activity of selected compounds against the HL60, SMMC-7721, A549, MCF-7, and SW480 cell lines.

\footnotetext{
These authors contributed equally to the work reported in this article.
} 


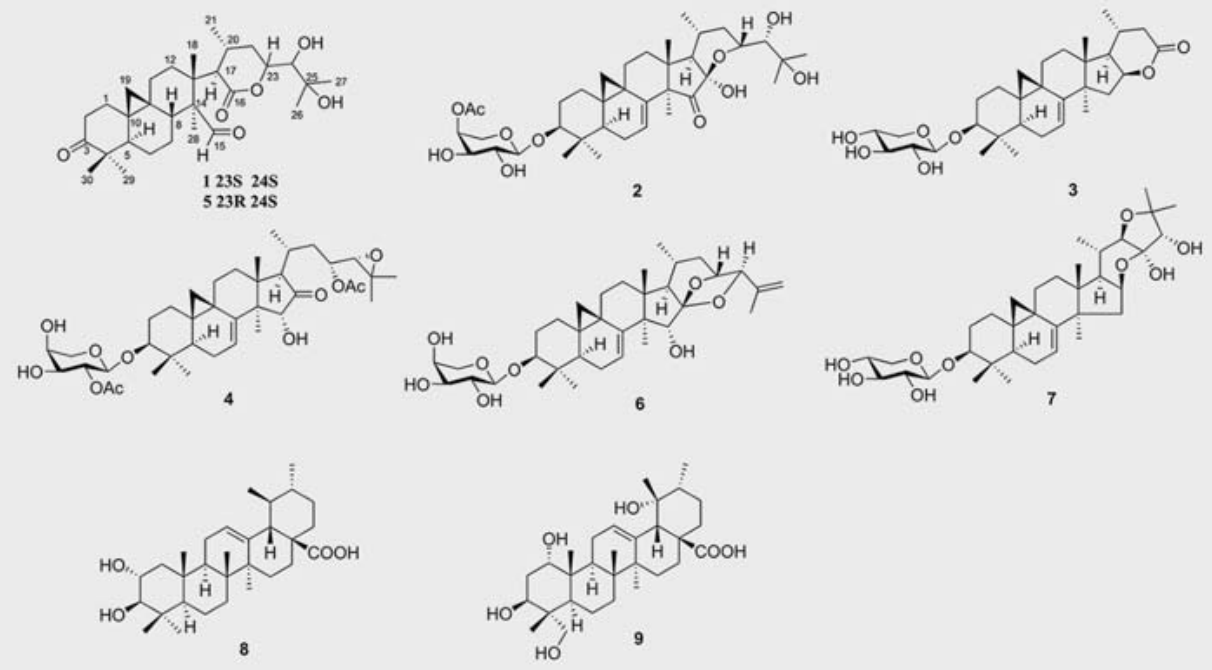

- Fig. 1 Chemical structures of compounds 1-9.

\section{Results and Discussion}

Compound 1 was obtained as white powder. The HRTOF-ESIMS ion signal at $\mathrm{m} / \mathrm{z} 525.3180[\mathrm{M}+\mathrm{Na}]^{+}$(calcd for 525.3192) indicated the molecular formula $\mathrm{C}_{30} \mathrm{H}_{46} \mathrm{O}_{6}$ with eight degrees of unsaturation. Its IR spectra showed the presence of hydroxy $\left(3438 \mathrm{~cm}^{-1}\right)$ and carbonyl functional groups $\left(1707 \mathrm{~cm}^{-1}\right)$. The ${ }^{1} \mathrm{H}$ and ${ }^{13} \mathrm{C}$ NMR data for $\mathbf{1}$ ( $\vee$ Table $\mathbf{1}$ ) were similar to those of cimiheraclein $D(5)$ [6], and the slight difference indicated that compound 1 was a configurational isomer of 5 , which was confirmed by its 2D NMR spectra (HSQC, ${ }^{1} \mathrm{H}-{ }^{1} \mathrm{H}$ COSY, and $\mathrm{HMBC}$ ) ( $\bullet$ Fig. 2). The ROESY correlations of $\mathrm{H}-23$ to $\mathrm{H}-20$ and of $\mathrm{H}-20$ to Me-18 suggested a $23 R$ configuration in compound 5 , while the correlations of $\mathrm{H}-23$ to $\mathrm{H}-17$ and of $\mathrm{H}-17$ to Me-28 suggested a 235 configuration in compound 1 ( $\vee$ Fig. 3 ). In addition, the configuration of $\mathrm{C}-24$ in compound 5 was proposed to be $S$ by comparison of the chemical shifts and coupling constants of $\mathrm{H}-23(5.07, \mathrm{~d}, J=11.2)$ and $\mathrm{H}-24(3.76, \mathrm{~s})$ of 5 with $23 \mathrm{R}, 24 \mathrm{~S}$ configuration analogue [H$23(5.01, \mathrm{~d}, J=11.1 \mathrm{~Hz}), \mathrm{H}-24(3.70, \mathrm{~s})]$ [8]. The configuration at $\mathrm{C}-24$ in 1 was finally confirmed by molecular modeling, in which $\theta=73.8^{\circ}$ in $23 R 24 S$ configuration, $\theta=177.7^{\circ}$ in $23 R 24 R$ configuration, $\theta=168.9^{\circ}$ in $23 S 24 R$ configuration, and $\theta=61.1^{\circ}$ in 235 245 configuration (Fig. 10S, Supporting Information), the coupling constants of $\mathrm{H}-23$ and $\mathrm{H}-24$ were $10.9 \mathrm{~Hz}$ and $0 \mathrm{~Hz}$, so the configuration of C-24 in 1 was proposed as $S$ based on the function of dihedral angle and $3 / \mathrm{H}-\mathrm{C}-\mathrm{C}-\mathrm{H}$. Also, this was consistent with other 15,16-seco-cycloartane derivatives reported previously [510]. Therefore, the structure of 1 was established as 15,16-seco14-formyl-(23S, 24S)-16-oxohydroshengmanol-3-one ( $\mathbf{F i g . 1 )}$ and given the name cimiheraclein $\mathrm{E}$.

Compound 2 possessed a molecular formula of $\mathrm{C}_{37} \mathrm{H}_{56} \mathrm{O}_{11}$ based on its HRTOF-ESIMS ion signal at $\mathrm{m} / \mathrm{z} 699.3715[\mathrm{M}+\mathrm{Na}]^{+}$ (calcd for 699.3720). Its ${ }^{1} \mathrm{H}$ NMR spectrum displayed resonances for cyclopropane methylene protons at $\delta_{\mathrm{H}} 0.50(1 \mathrm{H}, \mathrm{d}, J=4.0 \mathrm{~Hz})$ and $1.03(1 \mathrm{H}, \mathrm{d}, J=4.0 \mathrm{~Hz})$ for $\mathrm{CH}_{2}-19$, six tertiary methyl singlets at $\delta_{\mathrm{H}} 1.06,1.27,1.35,1.61,1.62$, and 1.64 (each $3 \mathrm{H}, \mathrm{s}$ ), and one secondary methyl at $\delta_{\mathrm{H}} 1.14(3 \mathrm{H}, \mathrm{d}, J=6.3 \mathrm{~Hz})$ as well as an anomeric proton signal at $\delta_{\mathrm{H}} 4.86(1 \mathrm{H}, \mathrm{d}, J=7.6 \mathrm{~Hz})$, which is characteristic of a 9,19-cyclolanostane-type triterpene glycoside. The sugar obtained after acid hydrolysis was identified as L-arabinose by comparing its TLC mobility and specific rotation with those of a standard. Detailed analysis of its NMR data ( $\vee$ Table 2 ) indicated that $\mathbf{2}$ is a $16 \alpha$-hydroxyl dahurinol-type triterpene glycoside and is similar to cimidahuside $C$ [20]. The major difference was the acetyl substituent. The observed ${ }^{1} \mathrm{H}-{ }^{1} \mathrm{H}$ COSY correlation of $\delta_{\mathrm{H}}$ $4.28\left(1 \mathrm{H}, \mathrm{m}, \mathrm{H}-5^{\prime}\right)$ to $\delta_{\mathrm{H}} 5.26\left(1 \mathrm{H}\right.$, brs, $\left.\mathrm{H}-4^{\prime}\right)$ and the HMBC correlation between $\delta_{\mathrm{H}} 5.26\left(1 \mathrm{H}\right.$, brs, $\left.\mathrm{H}-4^{\prime}\right)$ and $\delta_{\mathrm{H}} 171.2(-\mathrm{OAc})$ indicated the acetyl group was located at C-4' of the sugar moiety ( $\triangleright$ Fig. 2). The significant ROESY associations ( $\bullet$ Fig. 3) of $\mathrm{H}-3 / \mathrm{H}$ 5 and $\mathrm{H}-20 / \mathrm{H}-17$ suggested a 3S, 23R configuration. The hydroxyl group at C-24 was confirmed as $S$ by comparison of the chemical shifts and coupling constant of 2 with those of cimidahuside $C$ [20]. Accordingly, the structure of 2 was determined as $16 \alpha$ hydroxyl-7(8)-en-dahurinol-3-O-[4'-O-acetyl]- $\alpha$-L-arabinopyranoside ( $\bullet$ Fig. 1) and named as cimiheraclein F.

Compound 3 was isolated as a white powder and found to have the molecular formula $\mathrm{C}_{31} \mathrm{H}_{46} \mathrm{O}_{7}$ on the basis of the HRTOF-ESIMS ion peak at $\mathrm{m} / \mathrm{z} 553.3138[\mathrm{M}+\mathrm{Na}]^{+}$(calcd for 553.3141). The NMR data ( $\triangleright$ Table 2 ) of 3 were very similar to those of $3 \beta, 11 \beta$-dihydroxy-24,25,26,27-tetranor-cycloart-7-en-23,16 $\beta$-olide 3-O- $\beta$ D-xylopyranoside [21] except for the absence of the hydroxy group at $\mathrm{C}-11$. The stereochemistry of 3 was determined from its ROESY spectrum ( $\triangleright$ Fig. 3$)$. The crosspeaks of $\mathrm{H}-3\left(\delta_{\mathrm{H}} 3.50,1 \mathrm{H}\right.$, $\mathrm{dd}, J=11.7,4.1 \mathrm{~Hz})$ with $\mathrm{H}-5\left(\delta_{\mathrm{H}} 1.26,1 \mathrm{H}, \mathrm{dd}, J=12.5,5.1 \mathrm{~Hz}\right)$ and $\mathrm{H}-16\left(\delta_{\mathrm{H}} 4.88,1 \mathrm{H}, \mathrm{m}\right)$ with $\mathrm{H}-17\left(\delta_{\mathrm{H}} 1.92,1 \mathrm{H}\right)$ and $\mathrm{CH}_{3}-28$ $\left(\delta_{\mathrm{H}} 1.06,3 \mathrm{H}, \mathrm{s}\right)$ indicated the $\beta$-orientation of the substituents at $\mathrm{C}-3$ and $\mathrm{C}-16$. Therefore, 3 was elucidated as $3 \beta$-hydroxy-24, 25 , 26, 27-tetranor-cycloart-7(8)-en-23,16 $\beta$-olide 3-O- $\beta$-D-xylopyranoside ( $\bullet$ Fig. 1 ) and given the name cimiheraclein $G$. 
- Table 1 The NMR data of compounds 1 and 5 ( $\delta$ in ppm).

\begin{tabular}{|c|c|c|c|c|c|}
\hline \multirow{2}{*}{$\begin{array}{l}\text { Posi- } \\
\text { tion }\end{array}$} & \multirow[b]{2}{*}{$\delta_{\mathrm{H}}^{b}$} & \multirow{2}{*}{$\begin{array}{l}5 \\
\delta_{\mathrm{H}}{ }^{b}\end{array}$} & \multirow{2}{*}{$\begin{array}{l}\text { Posi- } \\
\text { tion }\end{array}$} & \multirow[b]{2}{*}{$\delta_{C}^{c}$} & \multirow{2}{*}{$\begin{array}{l}5 \\
\delta_{C^{c}}{ }^{c}\end{array}$} \\
\hline & & & & & \\
\hline 1 & $\begin{array}{l}1.81^{a} \\
1.51^{a}\end{array}$ & $\begin{array}{l}1.82^{a} \\
1.53^{a}\end{array}$ & 1 & $32.2 \mathrm{t}$ & $32.2 \mathrm{t}$ \\
\hline 2 & $\begin{array}{l}2.73 \mathrm{~m} \\
2.37 \mathrm{brd}\end{array}$ & $\begin{array}{l}2.72 \mathrm{~m} \\
2.38 \mathrm{brd}\end{array}$ & 2 & $37.4 \mathrm{t}$ & $37.4 \mathrm{t}$ \\
\hline 3 & & & 3 & $215.2 \mathrm{~s}$ & $215.1 \mathrm{~s}$ \\
\hline 4 & & & 4 & $50.3 \mathrm{~s}$ & $50.2 \mathrm{~s}$ \\
\hline 5 & $1.82^{a}$ & $1.81^{a}$ & 5 & $45.4 \mathrm{~d}$ & $45.4 \mathrm{~d}$ \\
\hline 6 & $\begin{array}{l}1.36^{a} \\
1.01^{a}\end{array}$ & $\begin{array}{l}1.38^{a} \\
1.01^{a}\end{array}$ & 6 & $19.2 \mathrm{t}$ & $19.2 \mathrm{t}$ \\
\hline 7 & $1.42^{a} 1.16 \mathrm{~m}$ & $\begin{array}{l}1.39^{a} \\
1.16 \mathrm{~m}\end{array}$ & 7 & $22.0 \mathrm{t}$ & $22.0 \mathrm{t}$ \\
\hline 8 & $2.47 \mathrm{~m}$ & $\begin{array}{l}2.44 \mathrm{dd} \\
(5.27 .4)\end{array}$ & 8 & $39.2 \mathrm{~d}$ & $38.9 \mathrm{~d}$ \\
\hline 9 & & & 9 & $22.1 \mathrm{~s}$ & $22.0 \mathrm{~s}$ \\
\hline 10 & & & 10 & $25.7 \mathrm{~s}$ & $25.7 \mathrm{~s}$ \\
\hline 11 & $1.64^{a} 1.54^{a}$ & $1.64^{a} 1.54^{a}$ & 11 & $27.2 \mathrm{t}$ & $27.1 \mathrm{t}$ \\
\hline 12 & $1.62^{a} 1.41^{a}$ & $1.62^{a} 1.41 \mathrm{~m}$ & 12 & $32.7 t$ & $32.7 t$ \\
\hline 13 & & & 13 & $47.9 \mathrm{~s}$ & $47.5 \mathrm{~s}$ \\
\hline 14 & & & 14 & $55.2 \mathrm{~s}$ & $55.3 \mathrm{~s}$ \\
\hline 15 & $9.97 \mathrm{~s}$ & $9.94 \mathrm{~s}$ & 15 & $208.0 \mathrm{~s}$ & $207.6 \mathrm{~s}$ \\
\hline 16 & & & 16 & $175.5 \mathrm{~s}$ & $175.3 \mathrm{~s}$ \\
\hline 17 & $2.76 \mathrm{~d}(5.5)$ & $2.74 \mathrm{~d}(5.6)$ & 17 & $55.8 \mathrm{~d}$ & $55.5 \mathrm{~d}$ \\
\hline 18 & $1.56 \mathrm{~s}$ & $1.56 \mathrm{~s}$ & 18 & $18.4 \mathrm{q}$ & $18.7 q$ \\
\hline 19 & $\begin{array}{l}0.80 \mathrm{~d}(4.7) \\
0.24 \mathrm{~d}(4.7)\end{array}$ & $\begin{array}{l}0.80 \mathrm{~d}(4.7) \\
0.24 \mathrm{~d}(4.7)\end{array}$ & 19 & $21.9 t$ & $21.9 t$ \\
\hline 20 & $2.05^{a}$ & $2.06^{a}$ & 20 & $28.8 \mathrm{~d}$ & $28.8 \mathrm{~d}$ \\
\hline 21 & $1.02 \mathrm{~d}(6.4)$ & $0.99 \mathrm{~d}(6.2)$ & 21 & $25.7 q$ & $25.1 \mathrm{q}$ \\
\hline 22 & $2.57 \mathrm{~m} 1.82^{a}$ & $\begin{array}{l}2.07^{a} \\
1.90 \mathrm{~m}\end{array}$ & 22 & $35.0 \mathrm{t}$ & $37.0 \mathrm{t}$ \\
\hline 23 & $\begin{array}{l}5.02 \mathrm{~d} \\
(10.9)\end{array}$ & $\begin{array}{l}5.07 d \\
(11.2)\end{array}$ & 23 & $80.9 \mathrm{~d}$ & $78.9 \mathrm{~d}$ \\
\hline 24 & $4.00 \mathrm{~s}$ & $3.76 \mathrm{~s}$ & 24 & $79.1 \mathrm{~d}$ & $80.2 \mathrm{~d}$ \\
\hline 25 & & & 25 & $72.2 \mathrm{~s}$ & $72.7 \mathrm{~s}$ \\
\hline 26 & $1.59 \mathrm{~s}$ & $1.67 \mathrm{~s}$ & 26 & $27.7 q$ & $26.2 \mathrm{q}$ \\
\hline 27 & $1.62 \mathrm{~s}$ & $1.73 \mathrm{~s}$ & 27 & $28.0 \mathrm{q}$ & $29.5 q$ \\
\hline 28 & $1.53 \mathrm{~s}$ & $1.51 \mathrm{~s}$ & 28 & $14.8 \mathrm{q}$ & $14.6 \mathrm{q}$ \\
\hline 29 & $1.01 \mathrm{~s}$ & $1.01 \mathrm{~s}$ & 29 & $20.6 q$ & $20.6 \mathrm{q}$ \\
\hline 30 & $1.08 \mathrm{~s}$ & $1.08 \mathrm{~s}$ & 30 & $22.6 \mathrm{q}$ & $22.5 q$ \\
\hline
\end{tabular}

Compound 4 was also obtained as a white powder, and its molecular formula was $\mathrm{C}_{39} \mathrm{H}_{58} \mathrm{O}_{11}$ based on its HRTOF-ESIMS ion signal at $\mathrm{m} / \mathrm{z} 725.3877[\mathrm{M}+\mathrm{Na}]^{+}$(calcd for 725.3877), which corresponds to 11 degrees of unsaturation. The NMR spectrum of 4 clearly displayed the signals characteristic of a 9,19-cycloartanetype triterpene. Direct analysis of its NMR data ( $\bullet$ Table 2 ) indicated that compound 4 resembles a 23-O-acetyl-7,8-didehy-

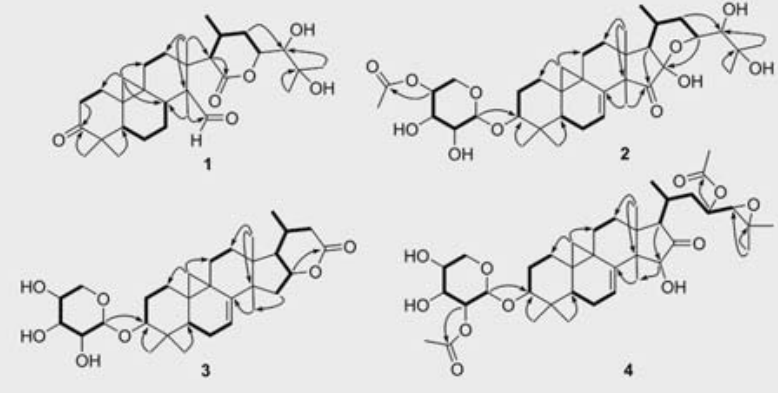

- Fig. 2 Major $\mathrm{HMBC}(\rightarrow)$ and ${ }^{1} \mathrm{H}-{ }^{1} \mathrm{H} \operatorname{COSY}(-)$ correlations of compounds 1-4.
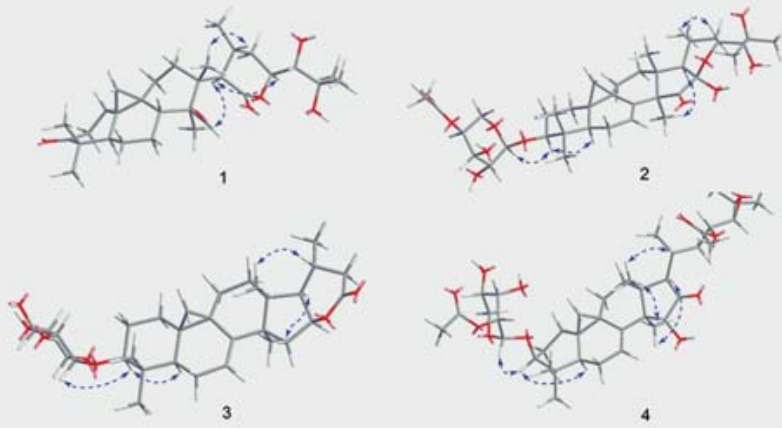

- Fig. 3 Key ROESY correlations of compounds 1-4.

droshengmanol-3-O- $\alpha$-L-arabinopyranoside [22] except for the presence of one additional acetyl group. The location of the acetyl group at C-2' ( $\bullet$ Fig. 2) was identified by the HMBC correlation between $\mathrm{H}-2^{\prime}\left(\delta_{\mathrm{H}} 5.58,1 \mathrm{H}, \mathrm{t}, J=8.3 \mathrm{~Hz}\right)$ and the carbonyl signal at $\delta_{\mathrm{C}}$ 170.4. The similarity between the chemical shifts and coupling constants of C-23 and C-24 in compound 4 with those of 23-O-diacetyl-7,8-didehydroshengmanol-3-O- $\alpha$-L-arabinopyranoside indicated the configurations of $\mathrm{C}-23$ and $\mathrm{C}-24$ were $R$ and $S$, respectively. Finally, the structure of 4 was confirmed as 23-O-acetyl-7 (8)-en-shengmanol-3-O-[2'-O-acetyl]- $\alpha$-L-arabinopyranoside, as shown ( $>$ Fig. 1).

The new compounds (1-4) were evaluated for their cytotoxicities against HL-60, SMMC-7721, A549, MCF-7, and SW480 cell lines ( $\vee$ Table 3 ). Compounds 1 and 2 did not show cytotoxic activity with $\mathrm{IC}_{50}$ value $>40 \mu \mathrm{M}$. Compound 3 showed weak activity against $A 549$ and MCF-7 cell lines with $\mathrm{IC}_{50}$ value 27.75 and $22.45 \mu \mathrm{M}$, respectively. Compound 4 also showed antitumor activity against the $\mathrm{HL}-60, \mathrm{~A} 549$, and MCF-7 cell lines with $\mathrm{IC}_{50}$ value $26.54,36.98$, and $21.34 \mu \mathrm{M}$, respectively.

The NMR, IR, UV, and HRTOF-ESIMS spectra of compounds 15 , as well as the dose-response curves of cytotoxic activity, are available as Supporting Information. 
- Table 2 The NMR data of compounds 2-4 ( $\delta$ in ppm, J in Hz).

\begin{tabular}{|c|c|c|c|c|c|c|}
\hline \multirow[t]{2}{*}{ Position } & \multicolumn{2}{|l|}{2} & \multicolumn{2}{|l|}{3} & \multicolumn{2}{|l|}{4} \\
\hline & $\delta_{\mathrm{H}}^{b}$ & $\delta_{C}^{c}$ & $\delta_{\mathrm{H}}^{b}$ & $\delta_{C}^{c}$ & $\delta_{\mathrm{H}}^{b}$ & $\delta_{C}^{c}$ \\
\hline 1 & $1.78 \mathrm{~m} 1.23 \mathrm{~m}$ & $30.7 t$ & $1.66 \mathrm{~m} 1.33 \mathrm{~m}$ & $30.7 t$ & $1.51 \mathrm{~m} 1.37 \mathrm{~m}$ & $32.4 \mathrm{t}$ \\
\hline 2 & $2.33 \mathrm{~m} 1.98^{a}$ & $29.8 \mathrm{t}$ & $2.35 \mathrm{~m} 1.97 \mathrm{~m}$ & $29.7 t$ & $2.27 \mathrm{~m} 1.91 \mathrm{~m}$ & $29.7 t$ \\
\hline 3 & $3.50 \mathrm{dd}(11.64 .0)$ & $88.8 d$ & $3.50 \mathrm{dd}(11.74 .1)$ & $88.5 \mathrm{~d}$ & $3.39 \mathrm{dd}(11.44 .1)$ & $88.6 \mathrm{~d}$ \\
\hline 4 & & $40.7 \mathrm{~s}$ & & $40.7 \mathrm{~s}$ & & $40.4 \mathrm{~s}$ \\
\hline 5 & $1.27 \mathrm{~m}$ & $42.2 \mathrm{~d}$ & $1.26 \mathrm{dd}(12.5,5.1)$ & $43.0 \mathrm{~d}$ & $1.31^{a}$ & $42.8 d$ \\
\hline 6 & $2.00^{a} 1.64^{a}$ & $22.1 \mathrm{t}$ & $1.87 \mathrm{~m} \mathrm{1.55^{ \textrm {a } }}$ & $22.2 \mathrm{t}$ & 1.97 m 1.62 m & $22.0 \mathrm{t}$ \\
\hline 7 & $6.70 \mathrm{~d}(6.3)$ & $117.3 \mathrm{~d}$ & $5.10 \mathrm{~d}(6.8)$ & $114.2 \mathrm{~d}$ & $6.14 d(7.2)$ & $115.3 d$ \\
\hline 8 & & $142.6 \mathrm{~s}$ & & $149.2 \mathrm{~s}$ & & $147.6 \mathrm{~s}$ \\
\hline 9 & & $21.8 \mathrm{~s}$ & & $21.3 \mathrm{~s}$ & & $21.8 \mathrm{~s}$ \\
\hline 10 & & $28.8 \mathrm{~s}$ & & $28.7 \mathrm{~s}$ & & $28.7 \mathrm{~s}$ \\
\hline 11 & $2.12 \mathrm{~m} 1.04 \mathrm{~m}$ & $25.1 \mathrm{t}$ & $2.06 \mathrm{~m} 1.15 \mathrm{~m}$ & $25.3 \mathrm{t}$ & $2.20 \mathrm{~m} 1.76 \mathrm{~m}$ & $25.5 \mathrm{t}$ \\
\hline 12 & $1.91 \mathrm{~m} 1.45 \mathrm{~m}$ & $31.6 \mathrm{t}$ & $1.63 \mathrm{~m} \mathrm{1.55^{a }}$ & $32.2 \mathrm{t}$ & $1.80 \mathrm{~m}$ & $33.8 \mathrm{t}$ \\
\hline 13 & & $40.7 \mathrm{~s}$ & & $44.4 \mathrm{~s}$ & & $41.2 \mathrm{~s}$ \\
\hline 14 & & $54.2 \mathrm{~s}$ & & $50.5 \mathrm{~s}$ & & $49.8 \mathrm{~s}$ \\
\hline 15 & & $212.5 \mathrm{~s}$ & $2.23 \mathrm{~m} 1.93 \mathrm{~m}$ & $42.9 t$ & $4.61 \mathrm{~s}$ & $81.2 \mathrm{~d}$ \\
\hline 16 & & $96.6 \mathrm{~s}$ & $4.88^{a}$ & $81.1 \mathrm{~d}$ & & $220.8 s$ \\
\hline 17 & $2.17 \mathrm{~m}$ & $57.8 d$ & $1.92^{a}$ & $54.9 \mathrm{~d}$ & $2.33 \mathrm{~d}(7.8)$ & $60.4 d$ \\
\hline 18 & $1.27 \mathrm{~s}$ & $23.3 \mathrm{q}$ & $1.07 \mathrm{~s}$ & $23.1 \mathrm{q}$ & $1.44 \mathrm{~s}$ & $19.7 q$ \\
\hline 19 & $1.03^{a} 0.50 \mathrm{~d}(4.0)$ & $28.1 \mathrm{t}$ & $1.07^{a} 0.47 \mathrm{~d}(3.9)$ & $28.7 \mathrm{t}$ & $0.86 d(4.0) 0.50 d(4.0)$ & $28.1 \mathrm{t}$ \\
\hline 20 & $1.97 \mathrm{~m}$ & $26.7 d$ & $1.93^{a}$ & $27.7 d$ & $2.17 \mathrm{~m}$ & $28.8 \mathrm{~d}$ \\
\hline 21 & $1.14 \mathrm{~d}(7.1)$ & $23.4 \mathrm{q}$ & $0.99 \mathrm{~d}(5.9)$ & $21.5 q$ & $1.25 d(6.5)$ & $20.1 q$ \\
\hline 22 & $2.45 \mathrm{~m} 1.69 \mathrm{~m}$ & $33.0 \mathrm{t}$ & $\begin{array}{l}2.46 \mathrm{dd}(14.72 .5) \\
2.28 \mathrm{dd}(14.712 .9)\end{array}$ & $39.1 \mathrm{t}$ & $2.91 \mathrm{~m} 1.74 \mathrm{~m}$ & $37.8 \mathrm{t}$ \\
\hline 23 & $4.66 \mathrm{dd}(11.75 .6)$ & $77.0 \mathrm{~d}$ & & $174.3 \mathrm{~s}$ & $5.45 \mathrm{~m}$ & $72.3 d$ \\
\hline 24 & 3.62 brs & $78.9 d$ & & & $3.08 \mathrm{~d}(8.4)$ & $65.6 \mathrm{~d}$ \\
\hline 25 & & $73.3 \mathrm{~s}$ & & & & $58.9 \mathrm{~s}$ \\
\hline 26 & $1.61 \mathrm{~s}$ & $26.8 \mathrm{q}$ & & & $1.28 \mathrm{~s}$ & $25.1 \mathrm{q}$ \\
\hline 27 & $1.62 \mathrm{~s}$ & $29.0 q$ & & & $1.45 \mathrm{~s}$ & $19.2 \mathrm{q}$ \\
\hline 28 & $1.64 \mathrm{~s}$ & $25.0 \mathrm{q}$ & $1.06 \mathrm{~s}$ & $27.1 \mathrm{q}$ & $1.30 \mathrm{~s}$ & $22.1 \mathrm{q}$ \\
\hline 29 & $1.35 \mathrm{~s}$ & $26.0 q$ & $1.37 \mathrm{~s}$ & $26.1 \mathrm{q}$ & $1.13 \mathrm{~s}$ & $25.8 \mathrm{q}$ \\
\hline 30 & $1.06 \mathrm{~s}$ & $14.5 \mathrm{q}$ & $1.08 \mathrm{~s}$ & $14.6 \mathrm{q}$ & $1.02 \mathrm{~s}$ & $14.4 \mathrm{q}$ \\
\hline $1^{\prime}$ & $4.81 \mathrm{~d}(7.3)$ & $107.9 \mathrm{~d}$ & $4.88^{a}$ & $107.9 \mathrm{~d}$ & $4.85 \mathrm{~d}(7.9)$ & $104.9 \mathrm{~d}$ \\
\hline $2^{\prime}$ & $4.42 \mathrm{t}(9.0)$ & $73.6 \mathrm{~d}$ & $4.08 \mathrm{~m}$ & $75.9 \mathrm{~d}$ & $5.58 \mathrm{t}(8.3)$ & $75.9 d$ \\
\hline $3^{\prime}$ & $4.29 \mathrm{~m}$ & 72.7 & $4.19 \mathrm{t}(8.8)$ & $79.0 d$ & $4.22^{a}$ & $76.6 \mathrm{~d}$ \\
\hline $4^{\prime}$ & 5.62 brs & 72.7 & $4.27 \mathrm{~m}$ & $71.6 \mathrm{~d}$ & $4.23^{a}$ & $71.7 \mathrm{~d}$ \\
\hline $5^{\prime}$ & $4.28^{a} 3.85 \mathrm{~m}$ & $64.8 \mathrm{t}$ & $4.40 \mathrm{dd}(11.25 .2) 3.77 \mathrm{~m}$ & $67.5 \mathrm{t}$ & $4.35 \mathrm{~m} 3.74 \mathrm{~m}$ & $67.5 \mathrm{t}$ \\
\hline $4^{\prime}-\mathrm{OAC}$ & $2.01 \mathrm{~s} \mathrm{~s}$ & $171.2 \mathrm{~s} 21.5 \mathrm{q}$ & & & & \\
\hline 23-OAC & & & & & $2.06 \mathrm{~s}$ & $\begin{array}{l}171.0 \mathrm{~s} \\
21.3 \mathrm{q}\end{array}$ \\
\hline $2^{\prime}-\mathrm{OAC}$ & & & & & $2.19 \mathrm{~s}$ & $\begin{array}{l}170.4 \mathrm{~s} \\
21.6 \mathrm{q}\end{array}$ \\
\hline
\end{tabular}


- Table 3 Cytotoxic activities $\left(\mathrm{IC}_{50}, \mu \mathrm{M}\right)$ of compounds $1-4$ against five human cancer cell lines. ${ }^{\mathrm{a}}$

\begin{tabular}{|l|c|c|c|c|c|}
\hline Compounds & HL-60 & SMMC-7721 & A549 & MCF-7 & SW480 \\
\hline 1 & $>40$ & $>40$ & $>40$ & $>40$ & $>40$ \\
\hline 2 & $>40$ & $>40$ & $>40$ & $>40$ & $>40$ \\
\hline 3 & $>40$ & $>40$ & $27.75(26.44-29.06)$ & $22.45(21.25-23.65)$ & $>40$ \\
\hline 4 & $26.54(25.51-27.57)$ & $>40$ & $36.98(35.88-38.08)$ & $21.34(20.37-22.31)$ & $>40$ \\
\hline DDP & $1.24(1.19-1.29)$ & $7.14(7.08-7.20)$ & $6.30(6.23-6.37)$ & $17.65(16.25-19.05)$ & $13.50(12.40-14.60)$ \\
\hline $\begin{array}{l}\text { a DDP (cisplatin) } \\
(n=3) .\end{array}$ & was used as a positive control. All data are present as the mean of IC 50 values with lower and upper $95 \%$ Cl from triplicate measurement \\
\hline
\end{tabular}

\section{Materials and Methods}

\section{General experimental procedures}

Optical rotations were recorded on a Horiba SEPA-300 polarimeter. UV spectra were acquired on a Shimadzu UV-2401A instrument. IR spectra were collected on Bruker Tensor 27 FTIR spectrometers with $\mathrm{KBr}$ pellets. NMR spectra were recorded on Bruker Avance III-600 spectrometers with tetramethylsilane as an internal standard at room temperature. HRTOF-ESIMS were recorded on an Agilent G6230 TOF spectrometer. TLC was performed on precoated TLC plates $\left(200-250 \mu \mathrm{m}\right.$ thickness, silica gel $60 \mathrm{~F}_{254}$, Qingdao Marine Chemical Inc.), and the spots were visualized by heating after spraying with $10 \%$ aqueous $\mathrm{H}_{2} \mathrm{SO}_{4}$. Semi-preparative HPLC was performed on an Agilent 1100 liquid chromatograph with a Zorbax SB-C18 (5 $\mu \mathrm{m}, 9.4 \mathrm{~mm} \times 250 \mathrm{~mm}, 3 \mathrm{~mL} / \mathrm{min})$ column.

\section{Plant material}

The aerial parts of $A$. heracleifolia were collected from Yichun County, Heilongjiang Province, China, in September 2012 and were identified by Prof. Zongyu Wang, Kunming Institute of Botany, Chinese Academy of Sciences. A voucher specimen (ZDSQ20120901) has been deposited at the State Key Laboratory of Phytochemistry and Plant Resources in West China, Kunming Institute of Botany, Chinese Academy of Sciences, China.

\section{Extraction and isolation}

The air-dried and powdered aerial parts of $A$. heracleifolia $(10 \mathrm{~kg})$ were extracted with $\mathrm{MeOH}(3 \times 30 \mathrm{~L} \times 24 \mathrm{~h})$ at room temperature to give a residue $(265 \mathrm{~g})$ after concentration under vacuum at $50{ }^{\circ} \mathrm{C}$. The extract was subjected to silica gel CC (200-300 mesh, $3 \mathrm{~kg}, 20 \times 150 \mathrm{~cm})$ and eluted with $\mathrm{CHCl}_{3}-\mathrm{MeOH}[100: 0(2 \mathrm{~L})$, $80: 1(5 \mathrm{~L}), 50: 1(5 \mathrm{~L}), 20: 1(8 \mathrm{~L})$, and $10: 1(8 \mathrm{~L})]$ to afford fractions A ( $3 \mathrm{~g}), B(5 \mathrm{~g}), C(2.5 \mathrm{~g}), \mathrm{D}(120 \mathrm{~g})$, and $\mathrm{E}(10 \mathrm{~g})$. Fraction B was divided into four sub-fractions (B.1-B.4) by RP-18 CC (20$45 \mu \mathrm{m}, 250 \mathrm{~g}, 5 \times 50 \mathrm{~cm}$ ) eluted with $\mathrm{MeOH}-\mathrm{H}_{2} \mathrm{O}$ (gradient from $50: 50$ to $100: 0,20 \mathrm{~L})$. Fraction B.3 (2.5 g) was subjected to repeated silica gel columns (200-300 mesh, $50 \mathrm{~g}, 5 \times 25 \mathrm{~cm})$ eluting with $\mathrm{CHCl}_{3}-\mathrm{Me}_{2} \mathrm{CO}$ (gradient from 20:1 to $5: 1,5 \mathrm{~L}$ ) and then purified by semi-preparative HPLC (eluting with $\mathrm{CH}_{3} \mathrm{CN}-\mathrm{H}_{2} \mathrm{O}$, gradient from $50: 50$ to $75: 25,3.0 \mathrm{~mL} / \mathrm{min}, 40 \mathrm{~min}, 210 \mathrm{~nm})$ to give compounds 1 (2 mg), 5 (4 mg), 8 (4 mg), and 9 (2 mg). Com- pounds 2 (9 mg), 3 (4 mg), 4 (8 mg), 6 (10 mg), and 7 (7 mg) were isolated from fraction C by silica gel CC (200-300 mesh, $50 \mathrm{~g}$, $5 \times 25 \mathrm{~cm}$ ) eluted with $\mathrm{CHCl}_{3}-\mathrm{Me}_{2} \mathrm{CO}(15: 1$ to $5: 1,5 \mathrm{~L})$ followed by repeated semi-preparative $\mathrm{HPLC}$ (eluted with $\mathrm{CH}_{3} \mathrm{CN}-\mathrm{H}_{2} \mathrm{O}$, gradient from $50: 50$ to $75: 25,3.0 \mathrm{~mL} / \mathrm{min}, 40 \mathrm{~min}, 210 \mathrm{~nm}$ ).

\section{Physicochemical properties of 1-4}

Cimiheraclein $E(1)$ : white powder; $[\alpha]_{D}^{25}=-69.25$ (c 0.08 , MeOH); UV (MeOH) $\lambda_{\max }(\log \varepsilon) 204$ (3.89) nm; IR (KBr) $v_{\max } 3440,2942$, 2871, 1700, 1632, 1457, 1380, 1032, $977 \mathrm{~cm}^{-1} ;{ }^{1} \mathrm{H}$ and ${ }^{13} \mathrm{C}$ NMR data $\left(\mathrm{C}_{5} \mathrm{D}_{5} \mathrm{~N}\right)$ see $>$ Table 1; HRTOF-ESIMS m/z $525.3180[\mathrm{M}+\mathrm{Na}]^{+}$ (calcd for $\mathrm{C}_{30} \mathrm{H}_{46} \mathrm{O}_{6} \mathrm{Na}, 525.3192$ ).

Cimiheraclein $F(2)$ : white powder; $[\alpha]_{D}^{25}=-10.49$ (c 0.15 , $\mathrm{MeOH}) ; \mathrm{UV}(\mathrm{MeOH}) \lambda_{\max }(\log \varepsilon) 204$ (3.78), 268 (3.07) nm; IR $(\mathrm{KBr}) v_{\max } 3440,2964,2872,1734,1632,1456,1383,1055$, $1001 \mathrm{~cm}^{-1} ;{ }^{1} \mathrm{H}$ and ${ }^{13} \mathrm{C}$ NMR data $\left(C_{5} \mathrm{D}_{5} \mathrm{~N}\right)$ see $>$ Table 2; HRTOFESIMS $\mathrm{m} / \mathrm{z} 699.3715[\mathrm{M}+\mathrm{Na}]^{+}$(calcd for $\mathrm{C}_{37} \mathrm{H}_{56} \mathrm{O}_{11} \mathrm{Na}$, 699.3720).

Cimiheraclein $G$ (3): white powder; $[\alpha]_{D}^{25}=-80.57$ (c 0.07 , $\mathrm{MeOH}) ; \mathrm{UV}(\mathrm{MeOH}) \lambda_{\max }(\log \varepsilon) 203(4.00) \mathrm{nm}$; IR $(\mathrm{KBr}) v_{\max }$ 3440, 2964, 2869, 1721, 1631, 1455, 1382, 1045, $969 \mathrm{~cm}^{-1} ;{ }^{1} \mathrm{H}$ and ${ }^{13} \mathrm{C}$ NMR data $\left(\mathrm{C}_{5} \mathrm{D}_{5} \mathrm{~N}\right)$ see - Table 2; HRTOF-ESIMS $\mathrm{m} / \mathrm{z}$ $553.3138[\mathrm{M}+\mathrm{Na}]^{+}$(calcd for $\mathrm{C}_{31} \mathrm{H}_{46} \mathrm{O}_{7} \mathrm{Na}$, 553.3141).

23-O-Acetyl-7(8)-en-shengmanol-3-O-[2'-O-acetyl]- $\alpha$-L-arabinopyranoside (4): white powder; $[\alpha]_{D}^{25}=-49.33$ (c 0.08 , MeOH); UV $(\mathrm{MeOH}) \lambda_{\max }(\log \varepsilon) 204$ (3.96) nm; IR (KBr) $v_{\max } 3434,2926$, 2853, 1738, 1631, 1459, 1379, 1044, $989 \mathrm{~cm}^{-1}$; ${ }^{1} \mathrm{H}$ and ${ }^{13} \mathrm{C}$ NMR data $\left(\mathrm{C}_{5} \mathrm{D}_{5} \mathrm{~N}\right)$ see $>$ Table 2; HRTOF-ESIMS m/z $725.3877[\mathrm{M}+\mathrm{Na}]^{+}$ (calcd for $\mathrm{C}_{39} \mathrm{H}_{58} \mathrm{O}_{11} \mathrm{Na}, 725.3877$ )

\section{Hydrolysis and identification of the sugar moieties in compounds 2 and $\mathbf{4}$}

Compounds 2 and 4 ( $2.5 \mathrm{mg}$ of each) were dissolved in methanol $(3 \mathrm{~mL})$ and refluxed with $1.0 \mathrm{~N} \mathrm{HCl}(2 \mathrm{~mL})$ at $90^{\circ} \mathrm{C}$ for $2 \mathrm{~h}$. After neutralizing with $1.0 \mathrm{~N} \mathrm{NaOH}$, the reaction mixtures were extracted with $\mathrm{CHCl}_{3}$, and the aqueous layers were concentrated under reduced pressure to give the monosaccharides, which had $R f$ values (EtOAc/ $\left.\mathrm{CHCl}_{3} / \mathrm{MeOH} / \mathrm{H}_{2} \mathrm{O}, 3: 2: 2: 1\right)$ and specific rotations $\left([\alpha]_{\mathrm{D}}^{20}+82.78, c 0.05, \mathrm{MeOH}\right)$ that were consistent with those of L-arabinopyranose (Sigma-Aldrich). 


\section{Biological assays}

Cytotoxic activity was investigated using five human cancer cell lines, human leukemia HL-60, hepatocellular carcinoma SMMC7721, lung cancer A549, breast cancer MCF-7, and colon cancer SW480 (Cell Bank of Chinese Academy of Sciences). Cells were cultured at $37^{\circ} \mathrm{C}$ in a humidified atmosphere of $5 \% \mathrm{CO}_{2}$ in RPMI1640 medium (HyClone) supplemented with 10\% (v/v) FBS (HyClone) and dispersed in identical 96-well plates. Compounds were dissolved in DMSO and serially diluted in saline to give final DMSO concentrations below $1 \%$. Each tumor cell line was exposed to the test compounds at concentrations of $0.064,0.32,1.6,8$, and $40 \mu \mathrm{M}$ for $48 \mathrm{~h}$ with cisplatin (DPP; Sigma, > 98\%) as the positive control; cell viability was determined by MTT cytotoxicity assay by measuring the absorbance at $570 \mathrm{~nm}$ with a microplate reader (Bio-Rad 680) [23]. Three independent trials were conducted for each compound $(n=3)$. The $I C_{50}$ values and $95 \%$ confidence interval $(\mathrm{Cl})$ were estimated using GraphPad Prism 6.

\section{Supporting Information}

The NMR, IR, UV, and HRTOF-ESIMS spectra of compounds $1-5$, as well as the dose-response curves of cytotoxic activity, are available as Supporting Information.

\section{Acknowledgements}

This research work was supported by the Autonomous Deployment Project (KIB2017010) of Kunming Institute of Botany, CAS, Program for National Natural Science Foundation of China (No. U1132604 and 81302670), and the Major Program of CAS (No. KSZD-EW-Z-004-01).

\section{Conflict of Interest}

The authors declare no conflicts of interest.

\section{References}

[1] Chinese Pharmacopoeia Commission. The Pharmacopoeia of Chinese People's Republic. 2015 Edition. Beijing: China Medical Science Press; 2015: 73-74

[2] Liu Y, Wu Z, Li C, Xi F, Sun L, Chen W. Heracleifolinosides A-F, new triterpene glycosides from Cimicifuga heracleifolia, and their inhibitory activities against hypoxia and reoxygenation. Planta Med 2013; 79: 301-307

[3] Nian Y, Zhang Y, Chen J, Lu L, Qiu M, Qing C. Cytotoxic chemical constituents from the roots of Cimicifuga foetida. J Nat Prod 2010; 73: 93-98

[4] Li J, Yu Z. Cimicifugae rhizoma: from origins, bioactive constituents to clinical outcomes. Curr Med Chem 2006; 13: 2927-2951

[5] Nian Y, Wang H, Su J, Zhou L, Feng G, Li Y, Qiu M. Cytotoxic cycloartane triterpenes from the roots of Cimicifuga heracleifolia. Tetrahedron 2012; 68: 6521-6527

[6] Wang W, Nian Y, He Y, Wan L, Bao N, Zhu G, Wang F, Qiu M. New cycloartane triterpenes from the aerial parts of Cimicifuga heracleifolia. Tetrahedron 2015; 71: 8018-8025
[7] Yoshimitsu H, Nishida M, Nohara T. Three new 15, 16-seco-cycloartane glycosides from Cimicifuga rhizome. Chem Pharm Bull 2007; 55: 789792

[8] Nian Y, Zhu H, Tang W, Luo Y, Du J, Qiu M. Triterpenes from the aerial parts of Cimicifuga yunnanensis and their antiproliferative effects on p53n236s mouse embryonic fibroblasts. J Nat Prod 2013; 76: 896-902

[9] Bao N, Nian Y, Zhu G, Wang W, Zhou L, Qiu M. Cytotoxic 9, 19-cycloartane triterpenes from the aerial parts of Cimicifuga yunnanensis. Fitoterapia 2014; 99: 191-197

[10] Nishida M, Yoshimitsu H, Okawa M, Ikeda T, Nohara T. Two new 15,16seco-cycloartane glycosides from Cimicifuga rhizome. Chem Pharm Bull 2003; 51: 1215-1216

[11] Chen J, Li P, Tang X, Wang S, Jiang Y, Shen L, Xu B, Shao Y, Li G. Cycloartane triterpenoids and their glycosides from the rhizomes of Cimicifuga foetida. J Nat Prod 2014; 77: 1997-2005

[12] Lu L, Chen J, Song H, Li Y, Nian Y, Qiu M. Five new triterpene bisglycosides with acyclic side chains from the rhizomes of Cimicifuga foetida $\mathrm{L}$. Chem Pharm Bull 2010; 58: 729-733

[13] Dan C, Zhou Y, Ye D, Peng S, Ding L, Gross M, Qiu S. Cimicifugadine from Cimicifuga foetida, a new class of triterpene alkaloids with novel reactivity. Org Lett 2007; 9: 1813-1816

[14] Nian Y, Lu N, Liu X, Li D, Zhou L, Qiu M. Antiacetylcholinesterase triterpenes from the fruits of Cimicifuga yunnanensis. RSC Adv 2018; 8: 7832 7838

[15] Nian Y, Yang J, Liu T, Luo Y, Zhang J, Qiu M. New anti-angiogenic leading structure discovered in the fruit of Cimicifuga yunnanensis. Sci Rep 2015; 5: 9026

[16] Ma C, Kavalier A, Jiang B, Kennelly E. Metabolic profiling of Actaea species extracts using high performance liquid chromatography coupled with electrospray ionization time-of-flight mass spectrometry. J Chromatogr A 2011; 1218: 1461-1476

[17] Kusano A, Takahira M, Shibano M, Miyase T, Okuyama T, Kusano G. Studies on the constituents of Cimicifuga species. XXII. Structures of two new cyclolanostanol xylosides, cimiacerosides $A$ and B. Heterocycles 1998; 48: 1003-1013

[18] Taniguchi S, Imayoshi Y, Kobayashi E, Takamatsu Y, Ito H, Hatano T, Sakagami H, Tokuda H, Nishino H, Sugita D, Shimura S, Yoshida T. Production of bioactive triterpenes by Eriobotrya japonica calli. Phytochemistry 2002; 59: 315-323

[19] Gupta D, Singh J. Triterpenoid saponins from Centipeda minima. Phytochemistry 1989; 28: 1197-1201

[20] Liu Y, Chen D, Si ], Pan R, Tu G, An D. Studies on the chemical constituents from the aerial parts of Cimicifuga dahurica. Acta Pharma Sinica 2003; 38: 763-766

[21] Nishida M, Yoshimitsu H. Six new cycloartane glycosides from Cimicifuga rhizome. Chem Pharm Bull 2011; 59: 1243-1249

[22] Kusano A, Shibano M, Kusano G, Miyase T. Studies on the constituents of Cimicifuga species. XIX. Eight new glycosides from Cimicifuga simplex wormsk. Chem Pharm Bull 1996; 44: 2078-2085

[23] Monks A, Scudiero D, Skehan P, Shoemaker R, Paull K, Vistica D, Hose C, Langley J, Cronise P. Feasibility of a high-flux anticancer drug screen using a diverse panel of cultured human tumor cell lines. J Natl Cancer Inst 1991; 83: 757-766 